\title{
Malaise dans la colonisation. Pour une clinique de la délinquance juvénile en pays kanak
}

\section{Umberto Cugola}

\section{(2) OpenEdition}

1 Journals

\section{Édition électronique}

URL : http://journals.openedition.org/jso/7724

DOI : $10.4000 /$ jso. 7724

ISSN : $1760-7256$

\section{Éditeur}

Société des océanistes

\section{Édition imprimée}

Date de publication : 15 décembre 2017

Pagination : 221-238

ISSN : 0300-953x

\section{Référence électronique}

Umberto Cugola, « Malaise dans la colonisation. Pour une clinique de la délinquance juvénile en pays kanak », Journal de la Société des Océanistes [En ligne], 144-145 | 2017, mis en ligne le 15 décembre 2019, consulté le 15 mars 2021. URL : http://journals.openedition.org/jso/7724 ; DOI : https://doi.org/ 10.4000/jso.7724

\section{(c) (i) $\ominus$}

Journal de la société des océanistes est mis à disposition selon les termes de la Licence Creative Commons Attribution - Pas d'Utilisation Commerciale - Pas de Modification 4.0 International. 


\title{
Malaise dans la colonisation. Pour une clinique de la délinquance juvénile en pays kanak
}

par

\author{
Umberto CUGOLA*
}

\section{RÉSUMÉ}

Depuis plusieurs années maintenant, la Nouvelle-Calédonie s'alarme sur un mal-être de sa jeunesse : suicide, addictions, conduites à risques... et la délinquance juvénile, qui est le phénomène du moment au regard de l'actualité locale. Elle touche principalement les Kanak, ceux des tribus proches de Nouméa, ceux des quartiers de la ville aussi, mais pas que. Le phénomène met en difficulté le sens commun quand il tente de comprendre ce type de comportement. Car l'opinion publique tend à jeter l'opprobre sur une jeunesse qui en plus d'être en difficulté se voit chargée d'une responsabilité pleine et entière de ses actes. Or, sil existe une justice des mineurs, c'est bien parce qu'une part de cette responsabilité est tout autant celle de la famille et, avec elle, de la société. Nous développerons dans cette contribution l'idée que la délinquance juvénile en pays kanak est le symptôme d'une société calédonienne elle-même souffrante. C'est en quoi une clinique du sujet est à mener en lien étroit à une clinique du social pour écarter les évidences que donnent à voir le phénomène.

MoTs-CLÉs : délinquance, symptôme, clinique, sujet, subjectivation, enveloppe, collectif, domination, aliénation, identité, socialité primaire et secondaire

\section{Avant-propos}

Dans une contribution où l'écriture est académique, il n'est jamais aisé d'oser dire «je». Pourtant, dans cet avant-propos, en quelques lignes seulement, je vais signifier l'intérêt que je porte à la

\section{ABSTRACT}

For several years, New Caledonia has been worrying about its youth's malaise and discontent as exemplified in suicides, addictions, risky behaviour and juvenile delinquency, the latter being the salient phenomenon in the local news. This mainly strikes the Kanak, those living in tribes close to Nouméa, as well as those living within urban Nouméa, but not only. It is difficult to understand such behaviour and phenomenon from the perspective of common sense. Public opinion tends to condemn this youth which, along with being helpless is laden with complete responsibility for their acts. However, the very existence of special courts for the underage points to part of the responsibility being that of the family and of society. In this contribution, we will develop the idea that juvenile delinquency in Kanak country is itself the symptom of a suffering Caledonian society. This is why a clinical approach of the subject should be conducted in close association with a clinical approach of the social. This in order to get rid of some of the all too obvious interpretations of this phenomenon.

KeYwords: delinquency, symptom, clinical approach, subject, subjectivation, wrapping, collective, domination, alienation, identity, primary and secondary sociality

\footnotetext{
* Éducateur spécialisé, cadre pédagogique à l'IFrass (enseignement en socio-anthropologie autour des objets métiers du travail social) et missionné sur des partenariats de recherche avec le département des Sciences de l'éducation de l'Université Toulouse II, et sur un projet européen de recherche Cap sur l'inclusion scolaire, ucugola@gmail.com
} 


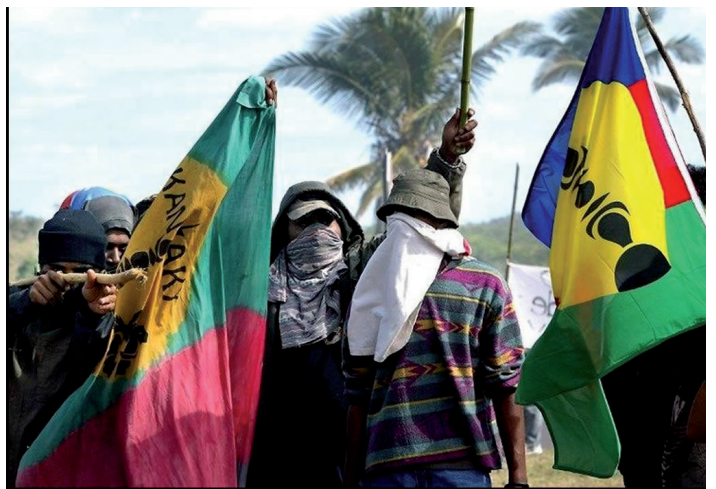

Рното 1. - Illustration du blog Calédospshère qui publie le 5 février 2016: "Déshérence et perte de repères: Une jeunesse kanak en perdition "! (C) Jerry Delathière)

- les tribus de La Conception et de Saint-Louis proches de Nouméa -, un mouvement partagé entre contingence et nécessité m’a propulsé loin des miens dans un exil qui est peut être avant tout intérieur. Mais si le cœur s'éloigne de ce qui est loin des yeux dans la passion amoureuse, c'est plus difficilement le cas quand on se détache de ses racines, tant il est impensable de mourir à ce qui nous a construit. Kanaky-Nouvelle-Calédonie peu importe ton nom, tes enfants t'aiment d'un amour jaloux et passionné, mais un jour ils auront la sagesse de te nommer comme tu es ou comme ils te rêveront. Tu es avec moi là où je suis et je te vois tous les jours sur la grande toile car le monde des réseaux dans lequel nous vivons n'est plus celui des relations épistolaires que nous avons tous deux connu. Tous les jours, mon pays, je vois partir nos vieux ou d'autres plus jeunes, nos enfants naitre, nos gens se marier, nos luttes politiques et syndicales perdurer... Et il y a un an, les Événements de SaintLouis avec leur tragédie sont venus bouleverser mon cheminement solitaire parce qu'ils ont mis en lumière la souffrance des miens et d'une jeunesse kanak qui ne va pas bien. Changer quelque chose à cela est impossible pour un seul homme, mais ne pas s'en soucier est inhumain... Alors, puisque je suis du dedans mais que je te vois du dehors, mon
Pays, voici ce que nous pourrions faire : mettre de la raison là où menacent les passions débordantes et les pulsions archaïques. Je préfère de loin la démarche inductive à l'abstraction mais à défaut d'être avec toi, commençons par penser ce en quoi consisterait un accompagnement éducatif et social de ta jeunesse. Peut être jetterons-nous ainsi les bases d'une autre perception du mal-être jeune, bases fondatrices qui sait, d'un mythe du retour au pays natal....

\section{Bref retour sur l'événement}

Le 29 octobre 2016 dans les environs du GrandNouméa ${ }^{1}$, le jeune William Décoiré (23 ans) trouve la mort, abattu par un gendarme après avoir refusé une opération de contrôle et foncé sur les militaires avec un véhicule. L'événement sera suivi d'une série d'exactions et de passages à l'acte par arme à feu ${ }^{2}$ à l'encontre des forces de l'ordre et des usagers de la route provinciale qui traversent régulièrement la tribu pour se rendre dans la capitale.

La gravité des faits entraînera une intervention du député calédonien Gomès à l'Assemblée nationale et une vive mobilisation du "Landerneau " politique et coutumier local. Il faudra près de six mois et des arrestations massives avant un retour au calme. Des moyens conséquents ont été annoncés par la ministre des Outre-mer, Ericka Bareigts, en faveur de la sécurité et de la prévention de la délinquance juvénile ${ }^{3}$. Un questionnement se pose ici : quels desseins idéologiques, conscients ou non, sous-tendent la réponse sociale et politique proposée ? Comment comprendre la délinquance juvénile dans certaines tribus kanak? Quelles sont les dimensions cachées de ce phénomène marginal? Étant entendu avec Michel Foucault ${ }^{4}$ que, quand on s'interroge sur ce que produit la marge, on parvient à mettre en évidence le cour politique du système et sa norme. Et au-delà, on tentera de saisir ce qui est en jeu dans le devenir de la société kanak et de la communauté de destin.

1. Avec Nouméa la capitale, les communes de Dumbéa, du Mont-Dore et de Païta composent l'agglomération urbaine du Grand-Nouméa où se trouve la tribu de Saint-Louis d'où était originaire W. Décoiré. Cet ensemble urbain qui englobe les tribus de Saint-Louis et La Conception a connu une explosion démographique massive ces dernières années : en 2009 deux Calédoniens (environ 160000 hab.) sur trois vivent dans l'agglomération urbaine (www.insee.nc Atlas démographique de la Nouelle-Calédonie).

2. Le 19 décembre 2016, meurtre d'un jeune Kanak de Saint-Louis, Ramon Noraro, sur fond de règlement de comptes entre bandes de la même tribu. Il y a aura aussi deux blessés dont un grave. Le 8 mars 2017, une jeune femme de 34 ans, Aurélie Marcias, est tuée à moto. Quatre mineurs de la tribu sont impliqués et poursuivis pour vol avec violences ayant entraîné la mort et non-assistance à personne en danger.

3. Annonce produite par la ministre en réponse au député Gomès à l'occasion des Questions au gouvernement du 17 novembre 2016 : la génération de contrat de développement 2017-2021 comporterait onze opérations "Actions pour la jeunesse " pour un montant de 15 millions d'euros de crédits d'État. Trois projets totalisant un montant de 4 millions d'euros seront spécifiquement dédiés à la tribu de Saint-Louis en faveur de l'insertion sociale et professionnelle de la jeunesse.

4. Avec notamment Surveiller et punir où le philosophe montre que la prison, modèle moderne de réponse pénale qui naît aux $\mathrm{XVIII}^{\mathrm{e}}$ et $\mathrm{XIX}^{\mathrm{e}}$ siècles, constitue l'aspect saillant d'une société désormais orientée vers le contrôle, la surveillance : une société disciplinaire. Il parle d'un assujettissement des individus à une norme travaillée par des systèmes institutionnels comme l'école, les usines, les hôpitaux, les casernes militaires... 


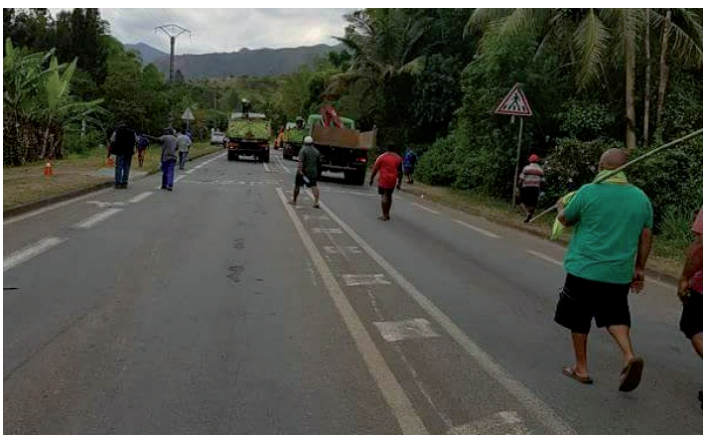

Рното 2. - Barrage sur la route provinciale Sud à hauteur de la tribu de Saint-Louis en 2016, à la suite du décès de W. Décoiré (@) www.outermer360.com)

\section{Quelle approche de la délinquance juvénile en question?}

Le droit recense trois types d'infraction en France : la contravention, le délit et le crime. Étymologiquement, le délinquant est donc celui qui commet une infraction à la loi par un délit. La délinquance qui nous intéresse ici est principalement la délinquance juvénile dont les actes se traduisent pour l'essentiel par des vols de voitures, des cambriolages, du trafic de cannabis, des agressions, des dégradations de biens privés ou publics. Les actes de délinquance ont massivement gangrené la vie sociale en Nouvelle-Calédonie avec une accentuation du phénomène ces trente dernières années. En 2011, dans un reportage sur une chaîne nationale d'information, Serge, chauffeur de taxi à Nouméa, l'avoue sans détour :

«Il y a trente ans vous laissiez votre voiture Place des Cocotiers avec les clés dessus et de l'argent, le lendemain vous retrouviez la voiture, les clés et l'argent... »"

Et à l'évidence, l'ère du développement (Leblic, 1993) incluse il y a trente ans dans les accords de Matignon (1988) puis de Nouméa (1998) s'est accompagnée de transformations sociales effrénées, terreau de phénomènes sociaux échappant aux systèmes de régulation et de contrôle social. Dans ses formes, la délinquance touche majoritairement des Océaniens et, en particulier, de jeunes Kanak. Il existe une délinquance dont les protagonistes vivent plutôt en territoire urbain, dans les quartiers populaires et les squats du Grand Nouméa (voir carte 1).

Et puis, il y a cette délinquance en lien avec les tribus, Saint-Louis, proche de Nouméa,

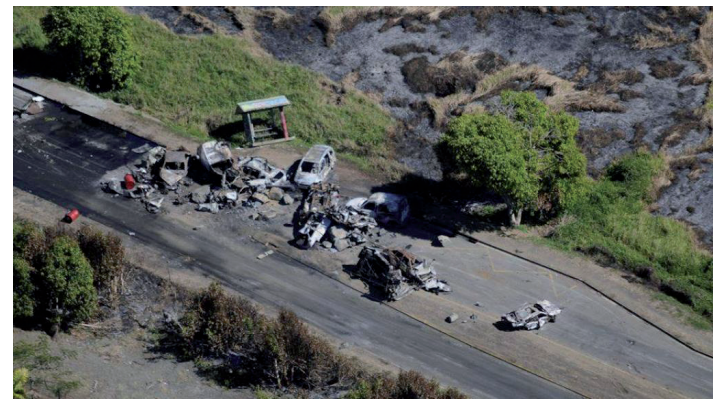

Рното 3. - En novembre 2016, à la suite du décès de W. Décoiré, manifestation d'un violent mécontentement : voitures volées et calcinées sur la route provinciale Sud qui traverse la tribu de Saint-Louis (@) http://observers.france24.com)

la capitale, étant la plus tristement célèbre. Mais sa réputation en la matière n'a pas à être enviée par les tribus des communes de Canala, Thio et Houaïlou. Les butins de cette jeunesse délinquante s'affichent sans inhibition sur les réseaux sociaux comme autant de prouesses, de trésors de guerre qui viennent alimenter une surenchère entre bandes. Et les réseaux sociaux participent et soutiennent une structuration de la délinquance en phénomènes de bandes dont l'organisation révèle une véritable société parallèle reliée à "La $400 »^{6}$. Dans ces bandes, il y a aussi des figures d'autorité, parfois même maternelles, des hiérarchies, une organisation territoriale adossée aux territoires coutumiers des aires linguistiques et des tribus. Il y a des rivalités et des coalitions entre bandes, des spécialisations par bande en fonction de catégories particulières d'actes délictueux (car-jacking, home-jacking, trafic de cannabis, "bastons", etc.). Il y a encore des logiques d'action comme ces raids menés par certains jeunes délinquants des tribus sur "Nouméa la Blanche" pour expier le crime colonial et la permanence de son injustice à l'endroit des Kanak.

Bref... les échanges à distance avec nos informateurs ne permettent pas d'aller plus loin que ce portrait évasif, mais, sur le plan socioanthropologique, il est certain que la délinquance néo-calédonienne est encore trop peu connue. La connaissance à produire à son sujet est d'autant plus pressante qu'elle est pour l'heure relativement épargnée par une connexion à des réseaux internationaux du crime organisé. Et la connaissance en question n'est pas à envisager dans le registre d'une sociologie répressive et policière dont nous ne doutons pas de l'utilité à condition

5. Reportage BFM tv à l'occasion de la visite du président Sarkozy en Nouvelle-Calédonie en août 2011. Certes, nous sommes conscients, avec Laurent Mucchielli (2014), de la méfiance qu'il faut garder à l'esprit vis-à-vis de la statistique policière comme des représentations véhiculées par les médias pour nourrir ou apaiser le sentiment d'insécurité. Il n'en reste pas moins significatif que la personne interrogée dans ce reportage évoque spontanément une période de trente ans renvoyant à la signature des premiers accords. Raison pour laquelle nous retenons ce témoignage.

6. Après « L'île de l'oubli », « La 400 » est le nouveau nom donné au centre pénitencier de Nouville (quartier de Nouméa). 


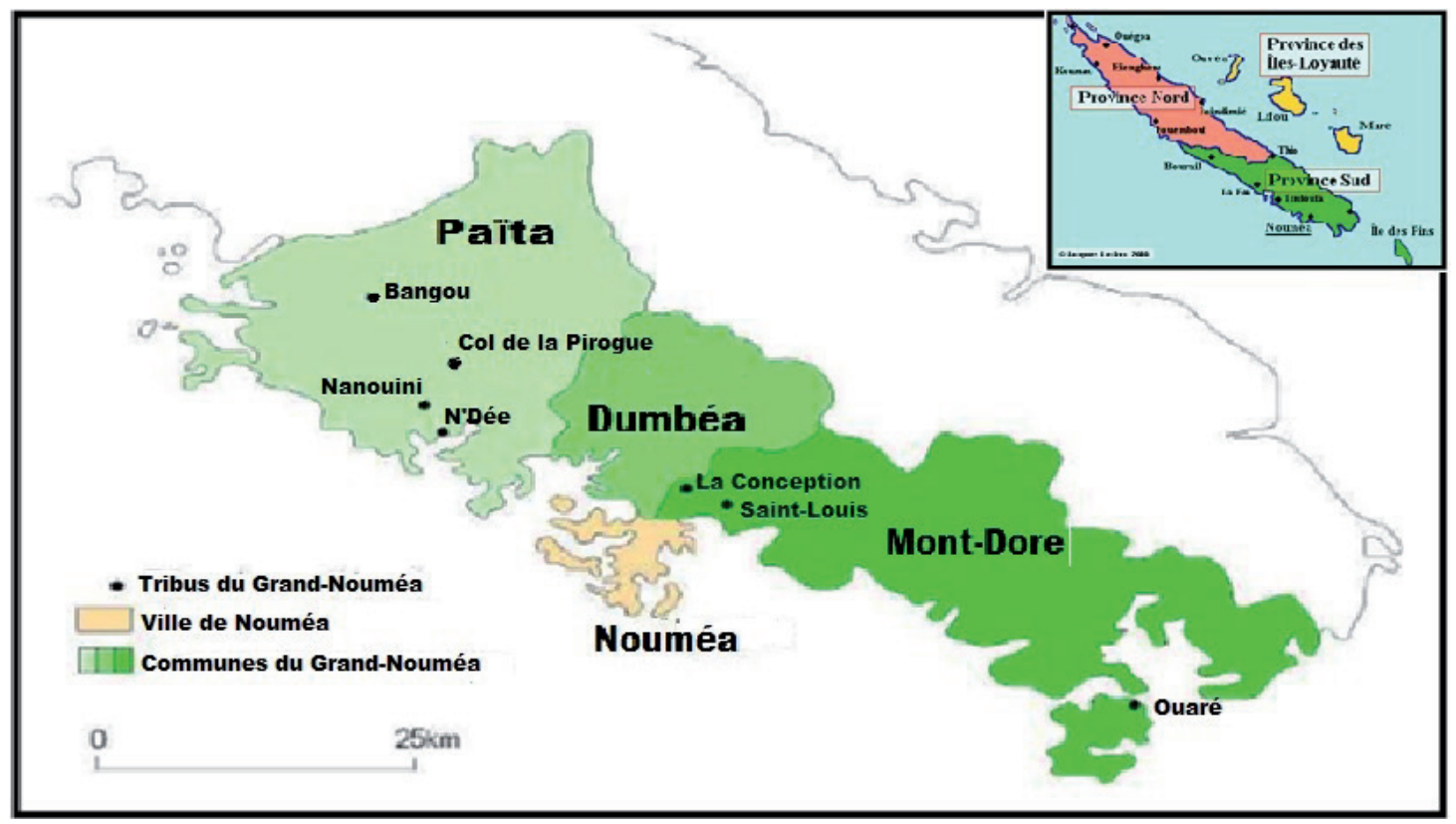

Carte 1. - Situation de la tribu de Saint-Louis au Mont-Dore, dans l'une des quatre communes du Grand Nouméa. Dans la même commune se trouve aussi La Conception, sa proche voisine et Ouaré à l'extrême sud. Avec N’Dée, Nianouni, Col de la Pirogue et Bangou dans la commune de Païta, le Grand Nouméa regroupe sept tribus parmi les 341 que compte la Nouvelle-Calédonie (carte principale, in Cugola, 2009)

de la situer à la place qui lui revient parmi les multiples approches possibles du phénomène qu'il nous faut donc esquisser. Commençons par traduire le phénomène par une question liminaire qui serait : comment interpréter la délinquance juvénile qui touche les Kanak de certaines tribus de Nouvelle-Calédonie?

Pour aller à l'essentiel, deux grandes familles d'approche théorique peuvent entreprendre de répondre à cette question. La première relèverait d'une certaine sociologie actionnaliste ${ }^{7}$ couplée à une discutable approche culturaliste. Selon elle, la délinquance serait une réponse des individus face à leurs possibilités limitées d'accéder à la vie économique et sociale ainsi qu’à la société de consommation. De telles limites seraient déterminées par un système familial et culturel intrinsèquement défaillant dans sa capacité à s'ajuster aux pratiques sociales de la modernité, dans sa propension à se laisser aller aux addictions en tout genre encourageant la délinquance. Dans un tel contexte, l'individu effectue un calcul rationnel, celui d'utiliser des moyens illicites pour obtenir un gain en évaluant une prise de risques. En somme une délinquance expliquée conformément au modèle de l'Homo xeconomicus mais sur le mode d'une déviance, celle-ci étant en germe dans un système culturel.

La seconde approche possible tendrait à montrer que la délinquance des jeunes Kanak résulte de difficultés dans le rapport des tribus à la société globale. Ce rapport étant un rapport de domination qui se traduit en premier lieu par une inégalité de principe sur le plan du niveau de vie au regard des standards des sociétés modernes (taux de chômage, niveau d'éducation, équipements en biens et services, etc.). C'est une domination qui par ailleurs exerce une pression assimilatrice sur l'organisation sociale coutumière en proieà des états d'anomie (perte de normes sociales), à une érosion des mécanismes de contrôle social et de contrôle de la déviance, à une crise multiforme de l'identité, à des dysfonctionnements dans la transmission... On peut ainsi avancer que le thème de la domination met en évidence un sentiment vécu d'inégalité dans un contexte de désorganisation sociale et que la délinquance n'est qu'une réponse sociale à un défaut d'ajustement entre dominants et dominés. Notre sensibilité va à cette approche, à ceci près que nous ne faisons pas des dominés de pures victimes considérant que leur domination est la condition même de leur émancipation et de leur devenir. Que ce soit par un État colonisateur ou par un Etat souverain et/ou indépendant, s'impose en dernière instance le principe d'une domination des territoires coutumiers par un pouvoir englobant exercé le cas échéant par ses propres élites. Loin d'être des victimes passives, les habitants des tribus réagissent et la délinquance est à comprendre selon nous comme le symptôme d'un dysfonctionnement dont il nous faut poser le diagnostic. En ce sens, l'approche clinique de la 
délinquance, que nous préconisons, est ici pertinente car la clinique, au chevet du souffrant alité, traite de ce qui dysfonctionne, des troubles et des carences. La notion de dysfonctionnement, rappelle Saül Karsz (2014: 120-121) a été introduite dans le champ de l'éducation, du travail social et de la psychologie par la sociologie et l'ethnologie d'inspiration fonctionnaliste. On considère comme dysfonctionnant ce qui se situe en dehors d'une norme, d'une normalité qui est cependant arbitraire puisque sa généalogie est impossible. Pourtant, dans la pensée $\mathrm{du}$ sens commun, nous sommes persuadés de son existence et avons tendance à clouer au pilori ce qui s'en écarte, telle la délinquance par exemple, que peu de gens aurait

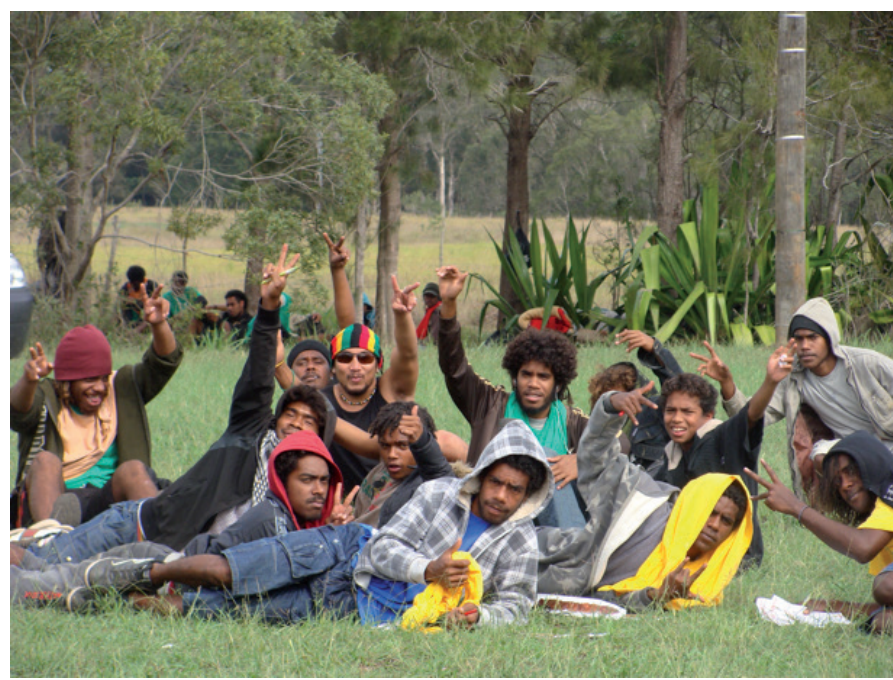

Рното 4. - Premier congrès de la jeunesse kanak en 2010 (http:// kanekarythm.centerblog.net)

l'audace d'envisager comme une subversion créatrice. Loin de nous l'idée de l'encourager et de nier sa tragédie, il s'agit plutôt de prendre le risque d'un renversement de perspective pour éclairer la délinquance juvénile kanak d'un diagnostic inédit pour la Nouvelle-Calédonie articulant une clinique du Sujet ${ }^{8}$ et une clinique du Social.

\section{Clinique et décentrement épistémologique}

La clinique que nous situons ici clairement dans le nouage de l'anthropologie et de la théorie psychanalytique s'inscrit dans le droit fil d'une réflexion critique sur le colonialisme et sur la dimension psychologique des rapports coloniaux et post-coloniaux. Elle emprunte aussi aux prises de position de la psychothérapie institutionnelle elle-même remaniée par Deleuze et Guattari dans l'Anti-CEdipe (Gabaron-Garcia, 2016). Ce positionnement, loin de faire le deuil d'une élaboration objectivante, affiche au contraire le matériau subjectif et les préoccupations éthiques qui fondent le mode de connaissance à produire à propos de la délinquance juvénile en pays kanak. C'est depuis un positionnement éthique et politique que cette contribution est énoncée, élaborée, proposée... car le contourner revient à verser dans l'intellectualisme.

Et nous sommes bien conscients que, succomber à la passion politique implique la gageure de céder à un activisme qui, comme l'intellectualisme, ne fait qu'obscurcir l'impensée du post-colonialisme. Il est donc de notre responsabilité de se prononcer pour une clinique du symptôme révélatrice de cette vie psychique du passé et des meurtrissures de l'histoire. Il ne s'agit pas de les attiser mais au contraire de penser le symptôme comme une résonnance en écho à la violence politique du pouvoir colonial: penser le symptôme et l'histoire pour panser les plaies de la mémoire. On peut affirmer en ce sens avec Lacan (1967) que l'« inconscient, c'est la politique".

Quel que soit le sens à produire, ce qu'on a à dire sur une société donnée et sur ses membres, l'éthique est première, elle précède l'épistémologie comme la reconnaissance précède la connaissance, rappelle Laplantine (2007 : 19-23) s'appuyant sur la phénoménologie d'Hegel : reconnaître l'Autre ${ }^{9}$ sur un principe d'équité, d'égalité, d' "éthicité " (comme dirait Hegel); une reconnaissance pas simplement par le langage et par les mots, mais aussi par les sens et par l'expérience, du regard mais pas seulement, celle du toucher des corps, de la communion des goûts, de la contagion des êtres et de leurs affects... La présence de cet Autre, de celui qu'on veut connaître, son être au monde, se pose comme une énigme pour le chercheur qui devrait se demander :

"Qu'est-ce que sa rencontre vient mettre en question dans mon existence?"

L'intersubjectivité précède donc le sujet dont on souhaite comprendre les pratiques, les comportements, les rapports sociaux... Les sciences humaines et sociales dans les colonies, au nom des Lumières ou d'un certain humanisme européen, s'enracinent dans une tradition où l'Autre est un objet de la connaissance. Elles

8. L'utilisation du terme de «Sujet » que nous faisons ici est inspirée pour l'essentiel par l'approche qu'en donne François Laplantine (2007). Nous argumentons ce choix à la fin de cet écrit.

9. «La majuscule à Autre reprend un usage popularisé par Lacan pour désigner l'altérité quand, radicale, elle ne se ramène pas à la distinction d'un autre semblable (minuscule). " (Sauret, 1993 : 19). 
étudient cet Autre à travers l'intelligible et les grands concepts ou thèmes qui se rapportent à lui : son organisation sociale, ses réseaux de parenté, ses rites, ses coutumes, son rapport au colonialisme, au développement, au destin commun... Or, l'intelligible de l'objet ne vaut que s'il est pensé en lien avec le sensible du sujet : les Kanak ne sont plus des objets à connaître mais bien des sujets de la connaissance, des sujets du langage, des sujets psychologiques et politiques résolument en quête d'un devenir émancipateur. Ce sont encore des sujets en interaction, socialisés dans leur contexte kanak avec un quotidien rythmé par l'alternance d'une vie sociale " ordinaire " et d'une vie en lien avec la coutume et ses cérémonies. Cela ne devrait jamais être d'eux dont parle le chercheur quand il produit de la connaissance mais de la relation qu'il a avec eux. On devine donc ici un premier décentrement épistémologique, exigeant une compromission éveillée du chercheur qui se voit inscrit dans un processus intersubjectif de coconstruction de la connaissance.

Le second décentrement épistémologique impliqué par la clinique est à comprendre à la lumière de l'ethnologie de Grégory Bateson qui étudie Les cérémonies du naven chez les Iatmul dans la région du Sépik en Papouasie Nouvelle-Guinée. Plutôt qu'une description à prétention exhaustive dans la tradition de monographies telles Les Argonautes du Pacifique occidental de Malinowski, Bateson amène une réflexion épistémologique sur un autre rapport aux données ethnographiques.

Il s'intéresse à la dimension émotionnelle du rituel et son approche sera reprise soixante ans plus tard par Houseman et Severi (1994) qui adoptent formellement une position clinique pour donner une interprétation à l'action rituelle. Celle-ci, avec le naven, consiste en une cérémonie qui célèbre et vient saluer un passage pour l'enfant toutes les fois où il accomplit un acte méritant la reconnaissance culturelle du groupe. À l'occasion de ce passage, Wau et Lava $^{10}$ mettent en scène des comportements et une communication complexe sur lesquels se concentrent nos auteurs :

«pour nous fournir une image sur la manière dont des facteurs écologiques et psychologiques s'articulent en une forme sociale. " (Houseman et Severi, 1994 : 192)

Traduit dans le sens qui nous intéresse ici, la délinquance juvénile est à comprendre non pas comme un rite - quoiqu' elle revête les apparats d'une demande de ritualisation du passage adolescent, nous le verrons -, mais comme un

phénomène social complexe que nous nommons symptôme dans le langage de la clinique et dont les mécanismes peuvent être saisis en travaillant à partir " des deux extrémités du continuum espritsociété " (Houseman et Severi, 1994). Il s'agit selon cette approche d'examiner un cas particulier de phénomène de déviance, un symptôme, pour saisirà travers lui un assemblage de facteurs psychologiques et de facteurs sociaux qui donne à voir un monde kanak dans son devenir contemporain.

\section{La délinquance au miroir d'une clinique du Sujet et d'une clinique du Social}

Si, de prime abord, on peut penser que la théorie psychanalytique (Zafiropoulos, 2003) ne s'intéresse qu'au cas - au sujet s'entend -, on peut rappeler que l'intérêt de Freud s'est d'emblée porté sur une clinique du social que l'on retrouve en particulier dans ses textes les plus connus en la matière: Totem et Tabou (1913), Psychologie des masses et analyse du Moi (1921) et Malaise dans la Culture (1929). Somme toute, l'idée est assez simple à comprendre : quand un patient s'allonge chez l'analyste pour évoquer son symptôme, il parle de son "moi » évidemment, mais un "moi " relié par une histoire à sa mère, son père, sa famille, ses amis, éventuellement ses collègues, etc., bref un " moi » en relation avec cet "Autre " de Lacan. Sans entrer dans le détail, on a bien cette intuition qu'en mobilisant la théorie psychanalytique, on peut rendre compte de la façon dont se déploie le développement psychoaffectif de l'enfant qui s'origine dans une relation

10. Terme Iatmul signifiant : «frère de sœur ». Peut s'appliquer de manière classificatoire, c'est-à-dire dans une parenté étendue. Lava signifie " enfant de sœur». 
à la mère avant de s'étendre à la famille restreinte puis élargie puis à la sphère sociale au sens global. Notre choix d'approcher l'objet - la délinquance en le situant dans ce mouvement qui s'étire depuis l'individu ${ }^{11}$, ou plutôt du sujet, au social n'est pas fortuit. On peut en effet s'intéresser à ses causes en se focalisant sur le jeune délinquant et sa famille pour y découvrir des carences et des défaillances dont à l'évidence ils seront seuls responsables.

Dans ce cas, les politiques sociales n'auront plus qu'à orienter leur action vers une médicalisation de l'intervention éducative et sociale afin de traiter la pathologie (la délinquance) du sujet. Ce qui, de surcroît, aura le mérite de donner un visage humain à l'autre réponse possible, pénale et judiciaire celle-là. Cependant, toute société est toujours plus ou moins inductrice des symptômes du sujet. Et il n'est pas infondé de s'attarder sur l'observation et l'écoute de ce que dit ce symptôme en paroles et en actes, car il y a là un gisement de connaissance sur une anthropogenèse, sur la grande question fondatrice de l'anthropologie ${ }^{12}$ et sur le monde social dans lequel baignent les sujets de la "normalité " et ceux du symptôme. En ce sens, le symptôme du sujet déviant, pathologique, marginal, délinquant, etc. est aussi un symptôme du social... Depuis la périphérie (des exclus, des marginaux...), ce symptôme dit des choses sur ce qui ne va pas au cœur de l'ordre social dominant :

"La clinique des manifestations symptomatiques à l'échelle du cas, du sujet donc, exprime dans le même temps l'esprit du temps et de la culture. " (Zafiropoulos, $2015: 11)$

Autrement dit, se focaliser sur une clinique du sujet en excluant la prise en compte du social et de la culture où il baigne, revient pour le système à refuser de se remettre en question. On ne saurait aborder la question de la délinquance juvénile en attribuant au sujet seul la responsabilité du phénomène. S’il est bien responsable de ses actes, aspect que l'intervention éducative et sociale devra mettre au travail avec lui, la société porte la responsabilité quant à elle d'avoir éludé l'accompagnement des mutations d'un ordre social et symbolique, garant du cadre familial d'éducation et de transmission.

\section{Du Sujet au Social : modélisation}

On positionnera ici un auteur classique dont la vision pénétrante servira de point de départ à notre argumentaire. Norbert Elias rappelle que :

" la structure des sociétés de notre temps a pour caractéristique d'accorder plus de valeur à ce par quoi les hommes se différencient les uns des autres, [...] à leur "identité du je", qu'à ce qu'ils ont en commun, leur "identité du nous"." (Elias, 1991 : 208sq.)

Scrutant dans les langues l'apparition des mots, le sens des notions et des concepts et leur évolution dans l'histoire, il en vient à établir qu'il était inimaginable dans le monde Antique qu'un individu s'affirme sans référence aucune à un " Nous ", à une "entité collective, que ce soit le clan, la tribu ou l'État..." (Elias, 1991 : 209sq.). Il ne s'agit pas là de déplorer l'affirmation d'un " Je " individualiste au dépend de la disparition d'un "Nous" communautaire dans les tribus kanak. Nous sommes plutôt enclins à considérer que le sujet de tout temps se construit et s'affirme en disant "Je " - ou non d'ailleurs - dans un rapport l'" Autre ", à plusieurs autres... bref à un "Nous». Signe d'une fragilité de notre époque qui n'épargne pas la société kanak, l'Homme moderne est en déséquilibre et souffrirait d'un pénible mal au "Je »-« Nous" (Dufour, 2016: 53-82). Et c'est ce rapport du "Je" au "Nous" qui nous intéresse, car c'est là qu'on cherchera à comprendre les mécanismes de la subjectivation, à l'articulation d'une clinique du Sujet et d'une clinique du social. C'est là au fond que se situent les failles offrant la jeunesse en proie à la délinquance. Puis, sur la base d'une conceptualisation du processus de subjectivation, nous ouvrirons sur des pistes à la fois de recherche et d'intervention clinique permettant d'aborder la délinquance dans les tribus.

Le "Nous" fondateur d'un "Je ", c'est au départ celui de la famille et même, plus fondamentalement encore, c'est le "Nous " de la dyade mère-enfant. Dans cet attachement primordial, pour reprendre les mots de John Bowlby (2002), les corps mêmes sont intriqués. L'enfant enveloppé dans le ventre maternel va ressentir les premières sensations d'une contenance. Puis, par le contact du "holding", par les manipulations du " handling" (Winnicott, 1969), la mère et l'environnement nourricier stimulent les sensations de la peau. L'enfant perçoit ces sensations par le toucher mais aussi par les autres sens, les odeurs, les sons, les goûts. Et il va symboliser ce qu'il perçoit comme étant les limites de son corps. À partir de là, il éprouve le sentiment d'une contenance psychique, le sentiment d'être enveloppé dans ce que Didier

11. Nous distinguons " individu » et « sujet " par rapport à une argumentation bien précise que nous développons à la fin de cet article. Toutefois, dans l'emploi que nous faisons dans ce qui suit du concept de subjectivation pour désigner un devenir-sujet, nous n'avons pas la place d'être plus précis pour situer avec Marcel Gauchet deux moments déterminants dans le développement de l'être humain : «l'individuation psychique ", "l'individualisation sociale "... puis la subjectivation à proprement parler (Gauchet, $2012: 13-28$ ).

12. "Qu'est-ce que l'Homme?". 
Anzieu (1985) nomme le "Moi-peau». Pour aller à l'essentiel, disons que l'environnement précoce de l'enfant, son père, sa mère, une forme de "Nous" des premiers âges donc, interagit avec la peau de l'enfant... et les sensations que cela lui procure sont symbolisées, entraînant l'élaboration d'une représentation de lui-même. C'est donc dans cet enveloppement primordial, familial, que l'enfant en vient à élaborer son "moi", c'est là que débute in fine le fameux processus de subjectivation.

Ensuite, cet enveloppement maternel, puis familial, de départ, s'il a été "suffisamment bon " (Winnicott, 1969), ne cessera de s'étendre avec un sentiment de continuité à des enveloppes psychiques diverses et plus larges : des enveloppes groupales, sociales et culturelles qui sont autant de collectifs, de " Nous » donc, que l'on trouve dans la famille élargie, le clan, la tribu puis à l'école, à l'université, dans l'univers professionnel... Ce mouvement du dedans vers le dehors n'est pas sans rappeler la description ethnographique de Margareth Mead (1963 : 66-71) chez les Arapesh montrant comment la mère met au travail les sensations de l'enfant dans un corps à corps avant de le sevrer avec bienveillance et de détourner progressivement sa sensibilité en direction des autres, humains, animaux, végétaux.

Nous mettons ici à profit la métaphore de l'enveloppement psychique remanié avec fécondité par Didier Anzieu et d'autres avec lui ${ }^{13}$, d'une part, pour situer le développement psychosocial du sujet qui nous intéresse (dans un rapport du "je " au "nous») et, d'autre part, pour repérer sur cette base ses déviances comme la manifestation d'une pathologie de l'enveloppe. Enveloppe qui s'étire depuis ses expériences fondatrices vers un univers culturel plus large ensuite qui le contient. L'idée d'une fonction structurante de l'enveloppe culturelle pour la vie psychique de l'individu est d'ailleurs reprise par Tobie Nathan $(1986)^{14}$. Elle est patente dans les situations de migration où la perte de l'enveloppe signe en même temps une perte sensorielle liées à des sons familiers, des odeurs et des goûts, des lieux, une langue, etc. qui met à mal la perception de soi qu'ont les personnes concernées.

Armés de cet éclairage conceptuel, on peut à présent proposer une lecture clinique de la délinquance juvénile émergeante en pays kanak. Cette lecture s'opère sur trois niveaux emboîtés

\section{entre eux :}

- l'enveloppe précoce du sujet dans sa famille, - l'enveloppe culturelle du sujet et sa famille, - puis l'enveloppe sociale de la modernité.

\section{L'enveloppe précoce du sujet dans sa famille}

Dans les échanges que nous avons pu avoir avec nos informateurs sur un profil du jeune délinquant, l'élément récurrent et marquant a été le constat de l'absence du père. Dans le pays paîci, l'un d'eux posait à regret le problème systématique émanant des enfants de mèrescélibataires $^{15}$. Pour d'autres, la cellule familiale est défaillante et présente une incapacité sévère à poser un cadre et une autorité éducative ferme telle qu'elle l'était autrefois. Et les réseaux sociaux donnent à voir un sens commun amer, regrettant la vigueur et l'efficacité de la bonne "trique " d'antan, pendant de la " tarte éducative " ${ }^{16}$ dans l'univers des éducateurs spécialisés. Quoi qu'il en soit, il semble que le desserrement d'une autorité éducative dans nos sociétés aille de pair avec le " déclin du père " dont la mort se serait produite il y a longtemps déjà selon l'auteur du Mythe de la horde dans Totem et tabou.

Examinons cette question du père à la lumière de la métaphore des enveloppes. En revenant à la théorie de l'attachement de John Bowlby en effet, il importe de préciser le poids de la fonction paternelle dans la séparation du lien fusionnel qui enveloppe physiquement puis psychiquement la mère et l'enfant. La mère est l'enveloppement primordial dans le creuset duquel, par un jeu d'identification projective et d'introjection (Ciccone, 2001), l'enfant élabore son contenant, le " moi-peau » chez Anzieu, qu'il investit ensuite d'un contenu, d'un sentiment d'être. Dans ce mouvement où l'intériorité de la mère et de l'enfant s'impliquent l'une l'autre, où les sentiments et les désirs ne sont jamais à l'abri d'errer sur les territoires interdits, le père est celui qui vient séparer. La molle étreinte dans laquelle s'enveloppe la dyade est prémunie contre la transgression ultime de l'interdit de l'inceste par l'intervention du père. Ce dernier ce faisant vient incarner la loi, l'ordre social et consolider le sentiment d'être en donnant une colonne vertébrale à l'enveloppe. La mollesse de l'étreinte maternelle alliée à la fermeté de la contrainte paternelle (Cugola, 2017) posent les conditions d'un enveloppement psychique dans

13. René Kaës et Didier Houzel notamment, à la suite des travaux principalement de Wilfried Bion et d'Esther Brick. Pour un historique concis sur le thème de l'enveloppe psychique, voir la conférence de la $15^{\mathrm{e}}$ journée de l'ARAGP (Lyon, janvier 2001) de Ciccone Albert (2001).

14. Le concept découle en fait des travaux de Devereux. L'enveloppe culturelle comme élément saillant de la structure du Sujet est une approche séduisante et féconde. Nous en ferons usage en restant toutefois attentif aux glissements culturalistes rendant le Sujet passif sans que l'on ne puisse rien analyser de sa singularité.

15. Phénomène aussi rapporté plus au nord de l'île par L. Enoka Camoui (2010).

16. En clair, cette idée commune que les enfants mériteraient d'être battus comme autrefois. Légitimée par le fait que nous avons tous survécu à la violence de nos aînés, ce qui a fait de nous les êtres fréquentables que nous serions devenus. 
une harmonie parentale où le sujet vient s'équilibrer entre deux pôles : la jouissance du côté de la mère, la loi du côté du père. Or, privé d'une éducation par la fonction paternelle ${ }^{17}$, le délinquant est celui qui n’a pas intégré la loi.

On peut aussi évoquer les défauts de contenance de l'enveloppe en regardant du côté maternel cette fois. À l'évidence, une mère privée du soutien du père et seule avec son enfant est insécurisée et ne bénéficie pas des meilleures conditions pour assurer une fonction contenante.

Il en va de même si, en situation de couple, elle est victime de maltraitance et de violence, argument souvent avancé par les associations militant en faveur de l'émancipation de la condition féminine en Nouvelle-Calédonie. Et, à juste titre, une mère dans l'incapacité de prodiguer un enveloppement sécurisant à l'enfant privera celui-ci d'un réceptacle à même de recevoir la vie pulsionnelle et émotionnelle éparse et désorganisée, due à son jeune âge. La fonction contenante est donc ce processus permettant de stabiliser les turbulences de la vie psychique de l'enfant et de les condenser en structures équilibrées et cohérentes ("moi", "surmoi ", "idéal du moi »). On le voit, le jeune enfant subissant sa vie pulsionnelle produit un comportement déviant qui, s'il n'est pas contenu par un dialogue constant avec la fonction contenante de l'enveloppe maternelle, est passible de perdurer sous une forme délinquante en avançant dans l'âge. Ce bouillonnement pulsionnel affecte par ailleurs le décrochage scolaire, souvent décrié localement comme cause de la délinquance, puisqu'il monopolise les capacités cognitives de l'enfant et le rend indisponible aux apprentissages.

\section{L'enveloppe culturelle du Sujet et sa famille}

Dans le cadre conceptuel proposé ci-dessus, sans l'avoir nommé expressément, on retrouve d'évidence un remaniement de la structure œdipienne avec sa triangulation : la mère, l'enfant et le père. Mais la combinatoire oedipienne doit à un premier niveau s'ajuster aux conditions sociales et culturelles dans lesquelles on tente de l'exprimer. Et, à un second niveau, cette causalité psychosexuelle ne saurait s'affranchir d'une causalité sociohistorique qui vient la compléter.

Au premier niveau en effet, s'il y a bien une dimension d'universalité du développement du sujet humain, on ne peut pas évacuer qu'il $\mathrm{y}$ a en même temps des conditions sociales et culturelles de l'œdipisme (Zafiropoulos, 2003) ${ }^{18}$. Et, à ce propos sur le terrain océanien, les travaux de Bernard Juillerat sont sans doute les plus illustratifs et les plus remarquables. Dans un hommage appuyé au regretté anthropologue, André Green (2010), Denis Monnerie et Pierre Lemonnier (2010) soulignent sa capacité à s'extirper des arguments rebattus pour donner à l'Edipe un sens plus ouvert, adossé à la rigueur reconnue de son ethnographie des Yafars en Papouasie Nouvelle-Guinée. On y découvre par exemple comment le conflit père-fils peut se dissimuler derrière une rivalité de l'aîné au cadet. $\mathrm{Au}$ second niveau, la prise en compte du social dans une approche clinique du sujet est une tentative pionnière que l'on retrouve chez Yoram Mouchenik (2004). L'auteur présente trois études de cas provenant des îles Loyautés (Maré et Ouvéa). Il adopte un point de vue anthropologique en situant l'enfant comme symptôme ${ }^{19}$ d'une histoire familiale et clanique qu'il s'attache à décoder en égrenant un parcours dans ce qu'il nomme une "étiologie locale».

La fécondité de ce concept, qui correspond à un savoir local sur les causes de la pathologie, lui permet de positionner l'enfant comme symptôme des projections familiales et/ou claniques. Il opère ainsi des incursions dans les conflits fonciers entre clans, dans des histoires d'adoption remises en question sur fond de fantasmes de dévoration d'un clan par un autre; autant de rapports conflictuels qui se cristallisent dans le symptôme de l'enfant. Nous retenons la démarche qui s'inscrit à la suite des apports de Georges Devereux sur la complémentarité entre l'activité psychique et son contexte culturel modelé par des dynamiques socio-historiques (Laplantine, 1972 : 42-44). Mais le point discutable de "L'enfant vulnérable » bien vu par Bernard Juillerat tient à ce que l'enfant y apparaît pour ainsi dire comme otage d'un mythe ou d'un "roman familial ». Il semble déterminé par son enveloppe culturelle alors qu'il est bien porteur d'une singularité qui se joue dans des processus oedipiens précoces où on doit lire sa relation à la mère et au père en prenant en compte le principe de la parenté classificatoire. Notre apport conceptuel se dessine là : situer le sujet et sa déviance, son symptôme, dans son enveloppe précoce, elle-même à saisir dans l'enveloppe culturelle d'un mythe ou d'un

17. Nous parlons bien de fonction paternelle et non de père car l'enfant peut très bien être éduqué par une mère seule sans difficulté aucune si celle-ci possède une capacité développée et équilibrée sur le plan de la bisexualité psychique et/ou si l'enfant dispose autour de lui de figures paternelles d'identification, comme c'est le cas en raison de la parenté classificatoire chez les Kanak.

18. L'auteur resitue dès les débuts de la psychanalyse une orientation donnée par Freud vers l'articulation d'une clinique du cas et d'une clinique du social. Un dévoiement se serait alors produit sous l'effet d'une approche américaine réduisant la théorie analytique à une théorie du « Moi » (l'individu) au détriment du Sujet, évacuant de fait la théorie du social. Celle-ci sera réactivée par Lacan qui s'appuie d'abord sur la sociologie durkheimienne avant d'avoir une révélation avec le structuralisme de Claude Lévi-Strauss.

19. Une pathologie sous la forme d'un handicap, maux de tête inexpliqués ou trouble du comportement. 


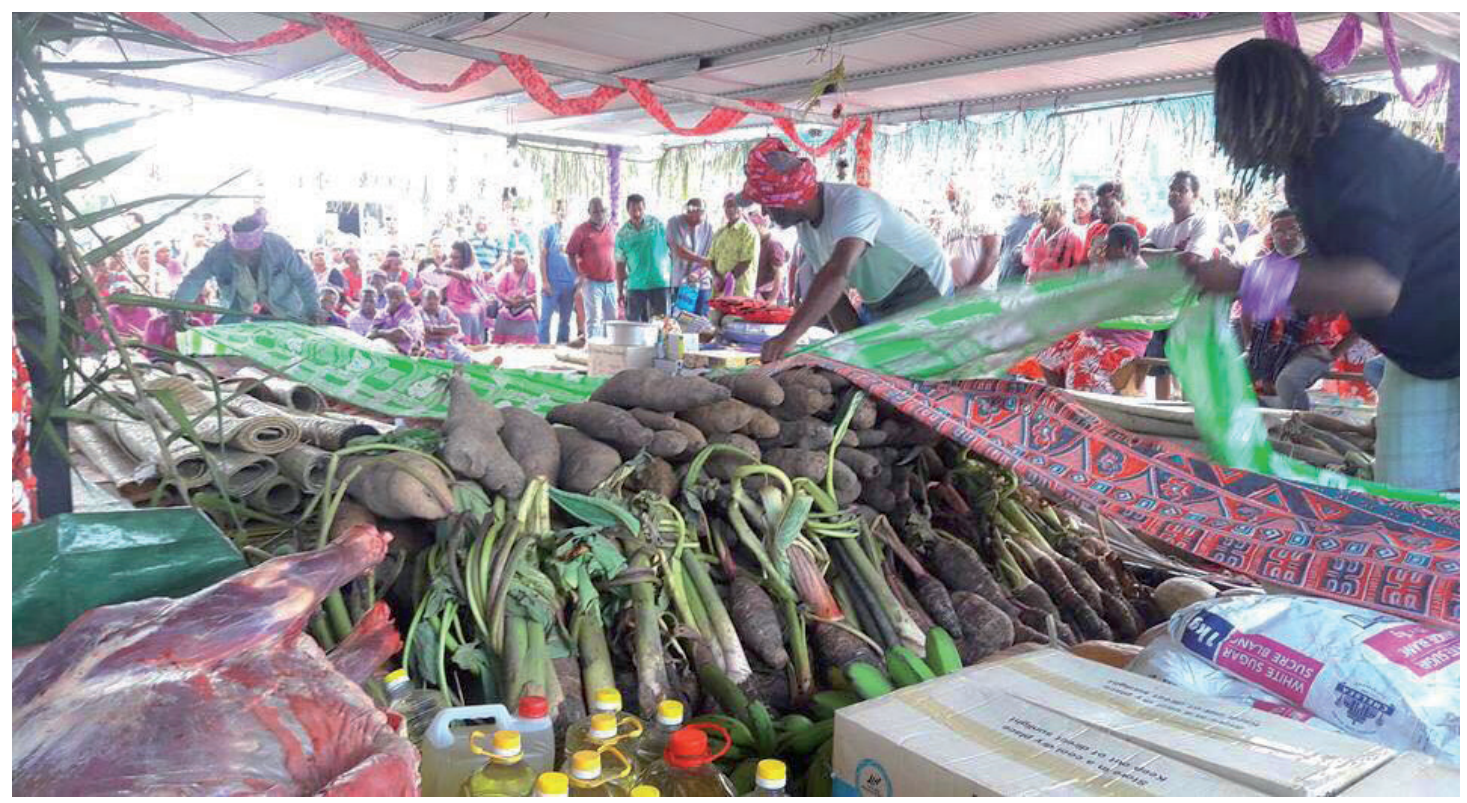

Рното 6. - Mariage coutumier, tribu de Saint-Louis, 2016 (@ page « Kanaky chez Nous », Facebook)

" roman familial ou clanique». On retrouve donc l'articulation du sujet au social que nous défendions au début de notre modélisation mais pour finir, nous proposons d'élargir l'enveloppe culturelle pour cette fois l'emboîter à une enveloppe sociale plus ample. D'une part, nous échapperions ainsi à l'écueil d'une dérive culturaliste, mais surtout nous prendrions en compte la dimension politique et les effets de domination sur l'enveloppe culturelle kanak.

\section{L'enveloppe culturelle kanak et l'enveloppe sociale de la modernité}

C'est à la lumière des apports de l'ethnopsychiatrie impulsée par Georges Devereux que nous aborderons le dernier étage de notre conceptualisation clinique de la délinquance. Avec lui, signalons sans détour que Seligman fut le premier à percevoir l'absence totale de structure psychotique dans les sociétés "authentiquement primitives » (Devereux, 1977 :212). Mais, préciset-il, faisant référence aux indiens d'Amérique qu'il a côtoyé, le fonctionnement psychotique apparaît chez les populations concernées dès lors qu'elles sont assujetties à " un violent processus d'acculturation et d'oppression "(Devereux, 1977 : 212). Adossé à la pensée de son aîné, Laplantine (1972 : 112) attribue à nos cultures modernes des propriétés "psychotisantes". Ce sont des cultures qui plongent les trajectoires individuelles dans un bain de complexité et les saturent de frustrations, de ruptures et de discontinuités entre ce qui a été acquis dans l'enfance et ce qui est exigé à l'âge adulte. La stabilité d'un cadre culturel - où le sujet est sensé puiser les ressources symboliques de sa subjectivation dans un rapport du " je » au " nous " - est sapée incessamment par la rapidité et la brutalité de transformations sociologiques portées par l'emballement des mécaniques économiques et financières. Dans ces conditions, ces sociétés produisent une « pollution psychotique » induisant des effets pathologiques sur des sujets insérés dans une "uniformisation monotone", cherchant par des "mécanismes régressifs de compensation" (déviance, délinquance, addictions, tentatives de suicide...) à amortir la pesanteur d'un réel devenu insupportable (Laplantine, 1972).

Les corps mêmes sont marqués par les effets de ces climats culturels. Qui, de retour d'un séjour «au Pays $»^{20}$, n’a pas été choqué en débarquant dans les aéroports parisiens, par les "visages figés " ${ }^{21}$ et mutiques se déplaçant dans un ballet d'automates à un rythme effréné et stéréotypé. Le contraste est d'autant plus saisissant si, vingt-quatre heures plus tôt, vous vous trouviez dans la douce chaleur d'un mariage coutumier avec son spectacle de couleurs, sa profusion de goûts et d'odeurs, enveloppé dans le rire des enfants et la bonhommie des convives.

Et c'est là que réside le grand intérêt de l'anthropologie et des chercheurs ${ }^{22}$ qui, depuis des années, s'attachent à rendre compte sur place de la richesse de ce que nous désignons par l'enveloppe culturelle des tribus kanak. Les "dynamiques cérémonielles » décrites par Denis Monnerie 
(2012) révèlent à travers "le transfert" et la circulation des objets de la coutume, la richesse et la profusion du lien social à différents niveaux (régional, local) et différentes étapes de l'existence (" cérémonies du cycle de vie »). La "chaîne opératoire cérémonielle" dans les coutumes supporte un rôle fondamental dans l'élaboration et l'actualisation des relations sociales. Elle pèse aussi dans un rapport au sacré et aux ancêtres, sans dogmatisme et avec le naturel de «l'homme religieux $»^{23}$. Elle suspend le temps ordinaire pour mieuxl'investir en sens en rappelant le sujetà l'ordre symbolique d'une filiation (ancêtres compris), d'une hiérarchie ou d'un positionnement entre clans et d'une modalité de rapport des hommes aux femmes. Ce qui n'est pas sans rappeler les trois anthropo-logiques de Georges Balandier (1974), concept éponyme de son livre, nous y reviendrons plus bas. Évidemment ce monde n'est ni angélique, ni indemne de conflits et de rivalités donc, qui parfois peuvent être régulés au cours de ces cérémonies. Mais celles-ci, préparées des mois durant avant l'événement " focal ", témoignent de l'intensité, du sens et de la vitalité du lien social que ce monde est en capacité de produire. Enveloppé dans un tel univers, le sujet ressent un "puissant sentiment de bien-être, d'accomplissement et de dépassement de l'expérience courante " nous dit encore Monnerie (2012). Celui qui a grandit dans un système communautaire reconnaitra qu'on y est immergé dans un tel sentiment de sécurité affective qu'en sortir pour se rendre à l'école ou dans les sphères professionnelles peut être vécu comme une agression. On y évolue sans doute sous la surveillance d'un collectif, mais celui-ci vous assigne une place et porte avec vous le sens à donner à votre existence, toujours négociable par ailleurs, avec plus ou moins de tensions. En tous les cas, le sujet enveloppé dans ces systèmes communautaires kanak n'est pas celui de notre modernité, abandonné à ses fantasmes et à ses pulsions, isolé dans une foule et un " être ensemble " de façade.

Or cette enveloppe culturelle subit la pression de facteurs extérieurs dont il nous faut à présent rendre compte. Pour ce faire, c'est dans la socioanthropologie d'Alain Caillé (2007) que nous avons mobilisé les outils conceptuels les plus pertinents pour actualiser la compréhension du rapport de domination succédant à la colonisation. Ce rapport de force-là pourrait être repensé - on ne le développera pas ici - dans un ordre mondial où une minorité de dominants cherche à fasciner une foule de dominés en leur inoculant des pseudo-désirs promis à une satisfaction éphémère sur un « Divin Marché » (Dufour, 2007).

\section{La force du Marché : socialité primaire et socialité secondaire}

Repositionnons ce que l'on cherche à comprendre : quelles pressions extérieures travaillent l'enveloppe du collectif des tribus au point d'y mettre le sujet en difficulté ? À la suite d'Alain Caillé et approfondissant ce que nous avions déjà mis en évidence dans nos travaux de thèse (" les effets du salariat sur le corps social d'une tribu urbanisée », Cugola, 2009 :262sq.), c'est la nature même du lien social qui change et s'immisce au cœur des tribus. Pour en comprendre les tenants, il faut distinguer les deux "registres de socialité " (Caillé, 2007: 86-87) qui structurent toutes sociétés.

D'abord, une "socialité primaire ", animée par un type de lien social moulé dans une dimension d'ordre symbolique faite d'idées, de croyances, de sentiments humains dont la sphère est celle de domaines aussi variés que la famille, la parenté, l'alliance, les réseaux de camaraderie, de solidarité, d'amitié et même d'amour ajoute A. Caillé (2007). C'est un collectif d'intersubjectivités, une enveloppe relationnelle dirons-nous fondée sur le principe d'un échange par le don, sur une obligation de donner, de recevoir et de rendre. Et, chez Caillé, la référence va clairement à Marcel Mauss qui identifie dans les sociétés traditionnelles cette modalité du don et du contre-don fondant un vivre ensemble.

La sphère de la "socialité secondaire " par contre est animée par un type de lien social à dimension utilitaire, dont l'aspect le plus saillant est dessiné par des rapports marchands. Dans cette sphère, le sujet est astreint à une fonction qui lui assigne une utilité sur un marché (consommateur), dans un système de production (salarié) ou dans un appareil d'État (salarié avec une mission de service public). Là, les rapports sociaux sont plutôt impersonnels et c'est la rationalité avec les notions de profit et d'efficacité qui guide les comportements. Si ces deux types de rapports sociaux sont plutôt imbriqués, il se trouve que nos sociétés modernes présentent cette particularité de provoquer " une disjonction croissante " entre eux tandis que les "autres sociétés" rechignent à les "désintriquer" (Caillé, 2007). Pour nous, les structures sociales, les clans, les familles, les tribus... du monde kanak sont profondément travaillées par une telle tension.

À un premier niveau, c'est dû au fait que le processus de stratification sociale, sous l'effet du salariat, de l'école et de l'urbanisation, décrit par des sociologues de l'orstom en 1985 s'est confirmé (Kohler, Pillon et Wacquant, 1985). La bourgeoisie mélanésienne, naissante il y a trente ans, est constituée aujourd'hui. On peut même

23. Donnée anthropologique fondamentale de l'humain chez Jung. 
pour aller plus loin et constater le développement d'un « capitalisme néotribal " (Babadzan, 2009: 211-249 ) en transférant sur le "Caillou " ce qui est observable chez nos voisins māori. Stimulé par les grands projets miniers et l'aménagement urbain, il introduit dans certaines tribus un niveau de modernité sans précédant. Après tout, qu'une élite éduquée et qu'un entreprenariat kanak s'affirment correspond bien à la trajectoire d'un rééquilibrage choisi par les leaders indépendantistes eux-mêmes.

$\grave{A}$ un second niveau, la mise en tension des structures sociales kanak est attisée par le projet néolibéral en Nouvelle-Calédonie comme ailleurs dans le monde, conduisant à une atomisation des collectifs dont la finalité n'est rien d'autre que la formation d'une société d'individus (Dufour, 2015) amputés de leurs liens de solidarité ("socialité primaire») afin d'accroître leur dépendance vis-à-vis du Marché. Le problème cependant tient à ce qu'une telle transformation a un coût, en premier lieu pour les enfants et les adolescents - Kanak ou non d'ailleurs -, en dérégulant les processus de subjectivation qui se jouent dans le rapport mutuellement impliqué du « Je» aux « Nous».

\section{La figure du Père, les grands récits et l'atomisation du collectif}

Le déclin de la figure du père et la fin des grands récits ont puissamment fragilisé la capacité des sociétés occidentales à produire du « Nous ». Pour certains cliniciens, ce déclin signe une mutation anthropologique majeure qui bouleverse l'économie psychique des sujets de manière inédite dans l'histoire (Gori, 2015: 211sq) ${ }^{24}$. Adaptons la perspective " lacanoïde " de Dufour (2016 : 55-56) pour rendre accessible notre argumentaire en commençant par rappeler que la psychose pour Lacan s'explique par la forclusion ${ }^{25}$ du Nom-du-père. À l'échelle individuelle : le sujet devient fou quand il est en relation à un père qui ne peut pas incarner une autorité, une antériorité et une altérité. Quand l'un de ces trois « $A$ » vient à manquer, le sujet devient fou car il n'a plus de point d'appui symbolique et imaginaire depuis lequel il peut ensuite se lancer dans le monde social en son nom, c'est-à-dire en disant "Je ».

À l'échelle collective à présent. Le collectif peut dire «Nous» quand il se reconnaît dans un Grand (sans tiret) Père, ou disons pour parler comme Lacan dans un grand Autre ou un Grand Sujet. Autrefois de Grands Sujets (Dufour, 2016) assuraient l'assemblage de petits sujets en un ensemble par des Grands récits : récit religieux, récit politique ou idéologique, et puis aussi le grand récit des Lumières qui fait de l'histoire de l'humanité une longue marche vers son émancipation. Mais, nous explique le philosophe Lyotard (1979), tous ces grands récits se sont effondrés, c'est-à-dire qu'ils ont cessé de faire sens pour les petits sujets. Or, au cœur de ces grands récits se tenaient une figure centrale, un grand père ou un grand sujet : un pape, un grand homme politique, un grand penseur du prolétariat, un maitre... Toutes ces figures possibles du grand sujet ont aujourd'hui perdu toute crédibilité et elles ne peuvent donc plus faire référence pour lier entre eux les petits sujets pour qu'ils forment un "Nous". Ce qui a remplacé les grands récits d'autrefois, ce sont aujourd'hui plein de petits récits publicitaires qui dictent aux petits sujets ce qu'ils doivent posséder pour dire « Je ». L'avoir a supplanté l'être.

Cette analyse vaut pour la société néo-calédonienne dans son ensemble et pour le monde kanak en particulier au sein duquel l'agonie de deux grands sujets mine la coalescence des petits sujets en clans, familles élargies, tribus..., bref en "Nous». Le premier grand sujet a disparu avec l'assassinat de pères du peuple : Jean-Marie Tjibaou, Éloi Machoro, Yeiwéné Yeiwéné, pour ne citer qu'eux. Ces personnages énonçaient des mythes, des discours, réalisant la synthèse de la diversité kanak en un seul et même pays. Ils portaient une parole politique fondatrice d'un grand récit de la nation et elle a été emportée avec leur disparition. Puis, comme dans toutes les démocraties modernes, le grand sujet de la classe politique aujourd'hui énonce une parole sans consistance parce que déconnectée des actes. Or, c'est bien en tressant des paroles et des actes que l'on donne sens à un récit qui permet à un « Je » de se reconnaître dans un "Nous » et de s'y positionner. À défaut, une parole sans actes, ça rend fou... Sommes-nous des sujets devenus fous? Dans l'éducation d'un enfant en tout cas, la chose est vérifiable.

La seconde figure paternelle dont le déclin mérite attention concerne les chefferies ou en tout cas un système d'autorité coutumière. On n'a pas la place ici d'entrer dans les dédales de l'histoire coloniale ayant conduit des légitimités coutumières à s'opposer dans des rivalités intes-

24. La perspective donnée par Gori vient bien entendu à la suite de Freud puis de Lacan (Lasch, 2000)... Elle rejoint aussi les approches de Melman (2009) et Lebrun (2007). Zafiropoulos (2009) est plus réservé sur cette question du déclin de la fonction paternelle dans le sens où, pour lui, il ne faut pas y voir une remise en cause radicale d'un ordre symbolique qu'on voudrait immuable, ce qui fait surtout problème dans la cure pour ce clinicien dont la critique va à ceux de ses collègues qui veulent à tout prix restaurer la figure du père auprès du Sujet au lieu de le soutenir en lui apprenant à vivre avec ce manque...

25. Terme juridique signifiant «fermé de l'intérieur ». Repris chez Lacan, la forclusion du Nom-du-Père désigne le cas limite du sujet psychotique fermé sur lui-même, sans possibilité d'accès à une " métaphore paternelle », à un "signifiant " de référence qui l'inscrit dans le langage et le lien social. 
tines. Mais on est rendu dans une situation aujourd'hui où de nombreuses tribus se retrouvent depuis plusieurs années sans chefferies. Au-delà des sempiternelles questions de légitimité entre chefferie administrative et chefferie coutumière, il y a urgence à poser qu'une chefferie pour une tribu est une figure d'autorité qui, incarnée, assure une fonction paternelle.

Si le dialogue, selon les trois " $\mathrm{A} »^{26}$ de Lacan, ne se produit plus entre une chefferie et sa jeunesse, la chefferie en vient à errer comme un « signifiant zéro ${ }^{27}$ passible de se charger de n'importe quel contenu symbolique. On serait alors dans la situation d'une jeunesse dans l'impossibilité d'associer au "signifiant zéro " - chefferie - un "signifié ", c'est-à-dire une figure qui viendrait incarner et rendre opérante dans la réalité sociale la fonction du père. C'est donc une situation où le «signifiant zéro " de la chefferie - le Nom-du-père chez Lacan - ne permet plus à la "pensée symbolique de s'exercer" (Zafiropoulos, 2009). Doiton en conclure avec Lacan à une forclusion du Nom-du-Père dont il dit qu'elle pose dès lors les conditions de la psychose, voire d'une psychose sociale ? Symptomatiques des mutations en cours dans le monde kanak, les récents événements de Saint-Louis ont montré dans toute leur tragédie le fossé qui s'est creusé entre les deux chefferies de cette tribu, entre les générations aînées et la jeunesse. De ce déclin de la figure du père en lien avec les chefferies, on peut tirer une conséquence qui se pose sur deux niveaux résonnant entre eux.

$\grave{A}$ un premier niveau, le malaise dans la relation du père au fils vient en même temps révéler une carence, un défaut dans l'anthropo-logique du passage de Georges Balandier (1974: 1013), soit ces logiques structurales inconscientes à l'œuvre dans toute société impliquée dans la production d'une vie sociale. Celle-ci, constate Balandier, quelle que soit la société concernée, n'est possible que si les hommes sont capables de mettre en œuvre des solutions symboliques et structurelles visant à réguler trois choses : le rapport des hommes aux femmes, le rapport entre les générations et le rapport des dominants aux dominés. Les solutions symboliques en question, ces anthropo-logiques donc, consistent en des systèmes de croyance, d'idées, de rites fabriqués pour lutter contre l'inachèvement du monde social en raison de sa complexité, pour lutter aussi contre sa vulnérabilité face au désordre menaçant qui, dans le même temps, le met en mouvement et le fait vivre. Et quand ces anthropo-logiques viennent à manquer, tels les rites de passage qui existaient en Europe aussi, tout se passe comme si un inconscient structural incitait les adolescents à organiser par eux-mêmes des solutions de passage (Lebreton, 2014) à travers leur conduites à risques (addictions, tentatives de suicide, déviance et passages à l'acte...). Celles-ci peuvent en effet être perçues comme des demandes d'épreuve que le collectif ne sait plus mettre en œuvre et ritualiser pour eux. Et cette dimension de l'épreuve est couramment observée durant la phase liminaire des rites initiatiques de passage dans les sociétés traditionnelles (Van Gennep, $1981)^{28}$. À travers l'épreuve liée à leurs conduites déviantes, c'est avant tout une reconnaissance que rechercheraient nos adolescents, un besoin d'être reconnu à une place d'adulte... de sujet en devenir. Autrement dit, les passages à l'acte peuvent tout autant être interprétés comme des actes de passage, soit des demandes de passage d'un âge vers un autre. C'est ce symbolisme du passage que les éducateurs spécialisés mettent en œuvre pour travailler avec les mineurs délinquants - ou non d'ailleurs ${ }^{29}$.

Le second niveau de conséquence entraîné par le déclin de la figure du père tient à ce que cette carence, vue dans une clinique du social, fait écho à la clinique du sujet. En effet, prenons le cas de l'adolescent délinquant présentant le profil dit des mères-célibataires ou tout autre cas d'adolescent porteur d'une problématique œedipienne déficiente sur le plan de la relation au père. La castration symbolique régulant à la fois l'interdit de l'inceste et l'intégration de la loi, donc l'accès au lien social, n’a pas pu se produire dans la triangulation odipienne infantile.

Par conséquent, au moment du passage adolescent, l'acte délictueux procède sur le plan social d'une tentative de reconstruction résonnant avec les dysfonctionnements de l'expérience précoce. Hélas, une telle tentative ne se joue fréquemment que sur un mode

\section{6. " Autorité, Altérité, Antériorité ", selon l'argumentaire évoqué ci-dessus.}

27. Lacan mobilise chez Lévi-Strauss ce concept pour soutenir sa théorie du Nom-du-père. Le « signifiant zéro " désigne une sorte d'attracteur présent dans tout univers symbolique et culturel qui potentiellement l'ordonne et le structure dès lors qu'il est appelé à être investi par le langage en un sens, un "signifié ».

28. Référence ici à la structure des rites de passage mise en évidence par Arnold Van Gennep : phase préliminaire de séparation, phase liminaire de réclusion en marge et phase post-liminaire dite d'agrégation et de retour.

29. Des dispositifs de rupture, comme les CER (centre éducatif renforcé) où j’ai exercé en tant qu'éducateur spécialisé, entrent dans ce registre. Ils mettent en œuvre des cadres symboliques permettant d'éprouver les trois phases du passage et de soutenir le processus de subjectivation chez l'adolescent. Ce ne sont pas des solutions toutes faites et miraculeuses mais, tout au plus, des espaces offrant à l'adolescent la possibilité d'éprouver une expérience structurante dont l'accumulation dans son parcours est susceptible de l'engager sur une voie résiliente. L'éducateur n’a pas de solutions infaillibles car son métier est bien cet impossible dont parlait Freud. Mais c'est parce qu'il ne renonce jamais à croire en sa commune humanité avec cet Autre en difficulté, qu'à défaut d'être possible, son métier a au moins un sens. Débattre, comme nous avons pu lire sur les réseaux sociaux, sur l'exhumation ou non de rites de passages qu’il faudrait réhabiliter pour lutter contre la délinquance juvénile chez les Kanak est trop simpliste pour penser une intervention éducative et sociale. 
pathologique ou pour le moins déviant puisque le sujet va s'attaquer à la figure paternelle ultime. Sous l'angle symbolique en effet, l'adolescent délinquant refuse la castration et exécute un meurtre du père en transgressant la loi. Il s'octroie alors la jouissance interdite en saccaparant les objets du Marché exhibés par la "mère-ville». Les choses seraient sans doute différentes pour le jeune Kanak si sa tribu ne subissait pas ce " processus d'intégration sociale " (Elias, 1991: 219) opérant un transfert du pouvoir (d'une figure symbolique du père donc) au profit de structures étatiques qu'il ne reconnait pas.

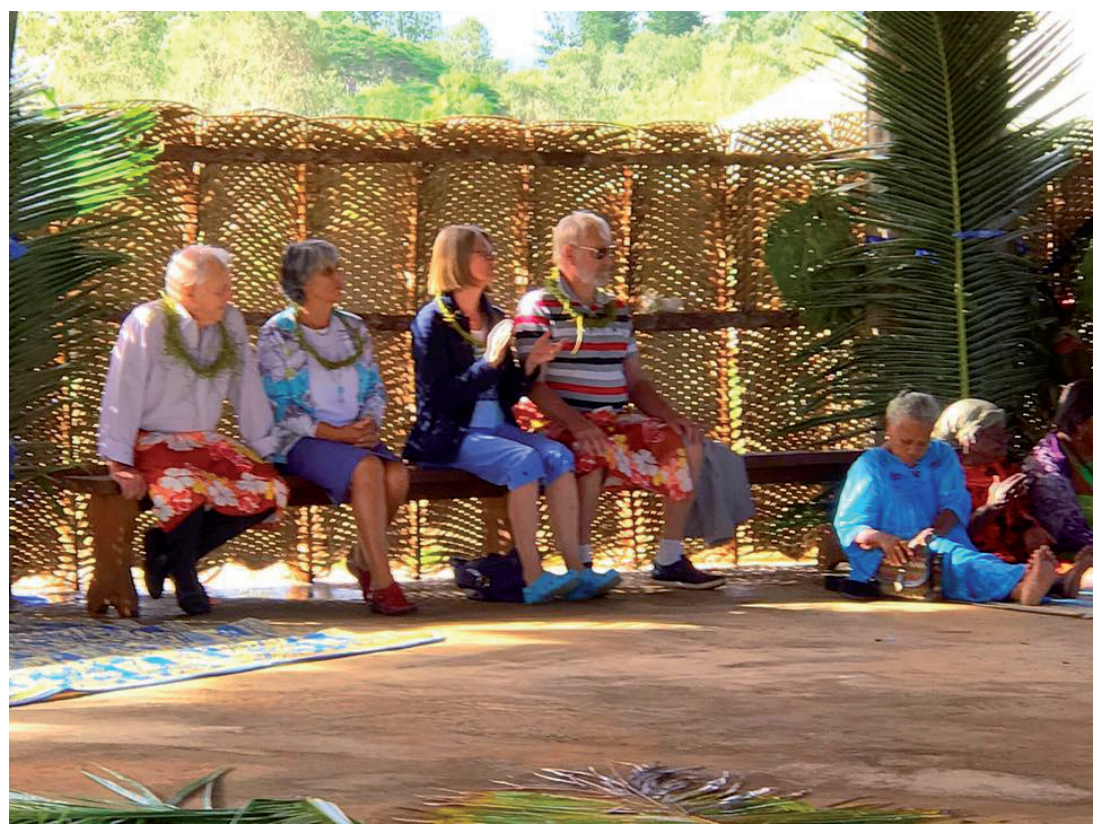

Рното 7. - Personnes d'origine européenne invitées à un mariage coutumier à Yaté au Sud de Nouméa en 2017 (@ page Facebook " Kanaky chez Nous »)

\section{Domination, aliénation et déviance}

Pour finir, nous allons examiner le processus articulant clinique du sujet et clinique du social, que nous venons de voir ci-dessus, sous un autre angle, par le prisme des effets de domination et d'aliénation hérités de la colonisation. Larrièreplan théorique qui soutient cette réflexion se réfere à l'œuvre de Frantz Fanon dont l'ethnopsychiatrie est beaucoup plus engagée que celle de Devereux ou plutôt de ses héritiers. Dans Peau noire masques blancs (1971: 108-110), il rapporte le cas du rêve d'un ses amis et patient qui, après un long parcours, parvient dans une salle où une assemblée de personnes de couleur blanche sont présentes. Puis, il finit par se voir blanc lui aussi. Fanon perçoit dans ce rêve la possibilité d'assouvir un désir inconscient refoulé du sujet, celui de s'identifier à l'homme blanc. Il y a là un complexe d'infériorité menaçant d'un effondrement sa structure psychique même. Mais surtout, si ce complexe existe, c'est parce que le sujet vit dans une société qui rend possible son existence et qui est même fondée sur elle. "Ce qui apparaît alors, nous dit Fanon, c'est la nécessité d'une action couplée sur l'individu et sur le groupe ». Et, à partir de là, dans la clinique, il s'agira d'orienter le patient non pas vers une mise à distance vis-à-vis de son désir de "lactation hallucinatoire", mais vers une prise de conscience des mécanismes inconscients qui le travaillent. Dès lors un choix devra lui être proposé : rester passif ou choisir l'action "à l'égard de la véritable source de son conflit psychique, c'est-à-dire à l'égard des structures sociales " mêmes qui produisent une telle aliénation $(\mathrm{Fa}-$ non, $1971: 108-110)$.

Dans la société calédonienne urbaine, on retrouve les mêmes principes avec un monde kanak pris dans un tiraillement contradictoire entre désir d'identification et repli identitaire. Les deux voies sont sans issues et nous souhaiterions nous attarder sur la seconde pour signifier la puissance avec laquelle elle parvient à produire une aliénation des Kanak par eux-mêmes. Sans revenir sur l'histoire qui a conduit la lutte nationaliste à être métabolisée en une reconnaissance culturelle (Babadzan, 2009), une telle transformation a pour rançon la menace d'un renforcement du repli identitaire. En outre, la culture kanak objectivée et institutionnalisée consacre un imaginaire et une idéologie de l'identité conquise de haute lutte.

Le sentiment de fierté, l'exaltation de cette identité est aussi irriguée par un inconscient collectif où sont refoulés les grandes meurtrissures, les humiliations, les traumas liés à l'histoire coloniale. Nous voyons deux registres de conséquence à l'idéologie identitaire ainsi produite. Le premier tient à un effet de communautarisme offrant au délinquant la possibilité d'être accueilli dans une matrice où il éprouve un sentiment de bien être et de sécurité affective. Encore une fois, si, dans son développement psycho-affectif se pose le problème de carences, de failles narcissiques, de fractures de l'enveloppe maternelle ou de dysfonctionnements dans la triangulation œdipienne, 
il va tenter une reconstruction sociale de sa personnalité, mais sur le mode de la pathologie. Car l'enveloppe communautariste lui donnera un sentiment illusoire de sécurité, un sentiment de confiance en soi, mais elle ne lui offrira pas les ressources nécessaires à l'expérience de l'altérité, à la régulation du passage de "l'endogène " à "l'exogène ", du «dedans " communautaire au "dehors" du social englobant. L'enveloppe est alors susceptible de devenir un foyer d'hébergement de groupes, de bandes délinquantes qui vont prendre en charge l'articulation du dedans au dehors mais sur un mode déviant. Et il n'est pas infondé d'émettre l'hypothèse que ces bandes viennent occuper une place de tiers symbolique laissée plus ou moins vacante par la figure paternelle des chefferies dont la fonction serait de séparer l'adolescent de l'enveloppe communautaire pour le propulser et l'inscrire dans un devenir en lien avec l'Autre, avec un ailleurs... bref avec notre commune humanité à tous.

Le second registre de conséquences pose le constat que l'idéologie identitaire est fondamentalement aliénante dans le sens où elle essentialise, elle fige l'individu, et non le sujet, dans un ensemble de traits culturels. La Boétie verrait là une Servitude volontaire qui ferme les horizons et engage une partie de la jeunesse kanak au mieux vers une introversion. Au pire, c'est au (non-)choix d'un repli identitaire xénophobe et vindicatif auquel elle est livrée, et qu'un discours populiste de gauche attise et manipule imprudemment. On ne compte plus les forfaits sur les réseaux sociaux affichant des voitures de luxe volées au nom de "Kanaky XXCRA "30. Le piège de Lillusion identitaire (Bayart, 1996) s'est refermé sur cette jeunesse délinquante, ce qui signale des structures sociales défaillantes dans la régulation de l'altérité. L'entre soi étant sans avenir, ce mode de fonctionnement du point de vue de la clinique du sujet est tout simplement pathologique. Il ne vaut pas dans un monde contemporain et, en particulier, dans une société calédonienne façonnée par des logiques métisses. Il y a certes un intérêt à faire usage de l'identité en tant que "catégorie de pratique " pour se positionner dans les relations du quotidien, pour raconter la rencontre et rendre compte de l'altérité... Mais pour penser le vivreensemble, il y a un écueil à attribuer à l'identité le statut d'une "catégorie d'analyse " car, ainsi, on en vient à considérer que les identités ethniques ont une existence réelle (Brubaker, 2001). Or, l'expérience du devenir-sujet est bien plus complexe qu'un ensemble de traits essentialisants. Dans le quotidien calédonien, ce devenir en acte est tout le temps déjà là : on voit des Kanak et des Wallisiens danser le Soamako ${ }^{31}$; on entend des Caldoches du Nord chanter au rythme du Kanéka ${ }^{32}$; un pasteur des îles peut être accueilli par des tempérances dans une paroisse de la Grande-Terre; une délégation wallisienne est accueillie coutumièrement à Ouvéa en mémoire d'une commune histoire précoloniale; ou encore des amis et des familles tahitiennes, javanaises, métropolitaines, accueillis coutumièrement lors de mariages traditionnels kanak...

Ce quotidien est d'une banalité en trompe l'œil car il est producteur d'interactions, d'intersubjectivités et de subjectivation selon des processus (identification, catégorisation, auto compréhension, créolisation...) permettant d'extraire l'analyse des horizons fermés pour se rendre "Au-delà de l'identité " (Brubaker, 2001), là où se trouve une commune humanité.

Pour finir, on reprendra à notre compte le raisonnement de François Laplantine (2007) en précisant que le sujet n'est pas l'individu, raison pour laquelle nous avons usé de ce concept tout au long de cet écrit. On est tenté de donner une essence à l'individu, de le définir comme une entité indivise avec un contenu de l'ordre d'une identité. Cette tentation par le concept d'identité de vouloir objectiver à tout prix la subjectivité humaine peut paraître suspecte voir dangereuse. Par exemple, l'individu peut être noir, blond, wallisien, caldoche, kanak, mais il peut aussi être une personne handicapée, un délinquant... Et voir les individus avec ces attributs identitaires, c'est les catégoriser, les diviser, les enfermer dans quelque chose et, parfois, les contrôler ou les dominer.

Un sujet, par contre, ne peut pas se définir a priori, il ne contient aucune substance car il est plutôt de l'ordre d'un devenir continu. C'est pourquoi il vaut mieux parler de processus de subjectivation, à la suite d'André Breton et de Michel Foucault (Laplantine, 2007 : 107). On peut tourner autour du sujet en le saisissant par plusieurs dimensions : le sujet politique (le citoyen), le sujet juridique (d'une culture minoritaire, d'une population particulière comme les personnes handicapées), le sujet psychologique (de l'inconscient du langage), le sujet de la connaissance qui est un sujet de la philosophie (le cogito ergo sum de Descartes par exemple). Nous ne développerons pas tous ces aspects ici mais il est patent que, quand on parle de sujet, on envisage l'homme, sa subjectivité, dans toute sa complexité. Le sujet n'est pas une substance indivise et fermée, enfermée dans une identité comme si c'était un objet. Un objet ne change pas, un objet est en proie à une impérissable domination tandis que le sujet est une relation, ouverte et dynamique, un potentiel en devenir.

30. NDLR. - Ce qui signifie "Kanaky vaincra » slogan apparu notamment sur les murs de Nouméa pendant et depuis les Événements.

31. NDLR. - Le soamako, danse d'origine guerrière wallisienne, qui correspond au haka māori.

32. NDLR. - Genre musical kanak ayant émergé pendant les Événements en 1984. 
C'est le sujet, dans la dialectique hégélienne du maître et de l'esclave, qui advient en se libérant de ce qui le domine, jamais l'objet... jamais l'individu et son identité donc.

C'est là un des grands enjeux pour le monde kanak aujourd'hui dans son attachement passionnel à sa culture (son ventre maternel). Le sujet, l'enfant kanak, est dépendant de cette enveloppe dans laquelle il a été formé, modelé, dans une subordination. Et s'affranchir de son enveloppe pour devenir soi est le premier passage, la première crise, la première émancipation dont le sujet doit faire l'expérience. Puis, si cette expérience fondatrice est réussie, le devenir-sujet consiste à se défaire sans fin de ce qui nous assujettit. Et, quand un peuple sait libérer ses enfants des passions exaltées de sa culture, alors, il a fini de subir la loi de ses oppresseurs.

\section{En guise de conclusion}

Quelle que soit l'enveloppe culturelle («normale " ou communautariste), l'enfant kanak baigne dans un univers dont il doit se séparer pour devenir lui-même. Il doit construire son expérience humaine dans un ailleurs, dans une singularité composant avec son héritage familial et culturel. Mais pour lui comme pour tous ceux qui ne sont pas de la culture dominante, le passage est toujours plus délicat. Car, du côté des dominés, les familles, les clans, les tribus, le collectif, le " Nous » donc... est puissamment fragilisé par des ruptures, des discontinuités que nous avons tentées de mettre en évidence. Pour autant, ce " Nous ", ce collectif, l'enfant kanak en a grandement besoin pour construire sa singularité de sujet. Il en a besoin pour se sentir aimé, pétri d'un sentiment de sécurité affective qui vienne nourrir son estime de soi. Sevré avec bienveillance du lien maternel, initié à la séparation par une figure paternelle, il capitalise alors suffisamment de confiance en soi pour avoir l'audace de se séparer dans une succession de passages depuis l'enveloppe fondatrice vers toutes les autres, celles qui l'ouvrent vers le monde.

Les rites et les systèmes symboliques de passage entre les âges et les espaces sociaux sont, avonsnous montré diffus, aujourd'hui. Certes, mais ils ne sont pas inexistants, dirait un éducateur, car s'il n'y a pas de solution $a d h o c$, il y a mille et une manières de se faire le passeur d'un enfant en difficulté. Dans une inlassable et ingrate répétition, l'éducateur invente, bricole des espaces symboliques permettant de remettre en scène les expériences précoces défaillantes en pariant sur une issue réparatrice et résiliente sur laquelle il reste conscient de n'avoir absolument aucune prise.

En Nouvelle-Calédonie aussi, l'éducation est en passe de devenir une science qui viendra expli- quer dans un discours objectivant ce que doivent être la famille, l'enfant, l'adolescent, la parentalité, bref... autant de catégories dont on sait la prétention normative et européocentrée. Et, en raison des éléments de langage dont il fait usage, l'écrit produit et proposé ici ne fait pas exception en déposant des outils conceptuels pour penser une pratique clinique locale. Nous serons prémunis contre une telle dérive en considérant dans la clinique du sujet une manière à chaque fois singulière (et donc subjective) d'éprouver une conduite déviante, un symptôme en lien avec le monde social. Ce symptôme, cet objet que l'on cherche à cerner entre une clinique du sujet et une clinique du social, est à la fois situé à l'articulation de l'anthropologie et de la psychanalyse, entre l'explicatif et le compréhensif. Nous avons voulu ici le rendre palpable, intelligible, mais on n'en épuise jamais le sens car il est par essence volatil en raison de la singularité des cas.

Cet écrit n'est rien d'autre qu'une contribution, suggestive plus que démonstrative, au débat sur un thème aussi touchant que celui d'un mal-être des enfants du Pays. Leurs fugues, addictions, tentatives de suicide, leurs passages à l'acte sont tout autant à comprendre comme une demande de passage et de devenir. Si la société ne sait plus y répondre, elle mérite tout autant d'être soignée que ceux qu'elle a la prétention d'enfermer ou d'éduquer...

\section{Remerciements}

À ma famille et mes amis en NouvelleCalédonie qui m’informent au quotidien sur le Pays et nourrissent le lien. Remerciements particuliers pour nos débats sur ce phénomène de la délinquance de nos jeunes à Erika et Maurice Jamet, Carole Pidjot, Jean-Claude Tutugoro, Christian Grochain, Teddy Dalmayrac et Anne Fochi. Enfin, aux lecteurs du JSO.

\section{BIBLIOGRAPHIE.}

Anzieu Didier, 1985 (nouv. Éd. 1995). Le Moi Peau, Paris, Dunod.

Babadzan Alain, 2009. Le spectacle de la culture. Globalisation et traditionalismes en Océanie, Paris, L'Harmattan.

Balandier Georges, 1974. Anthropo-logiques, Paris, PUf.

Bayart Jean-François, 1996. Lillusion identitaire, Paris, Fayard.

Bowlby John, 2002. Attachement et perte, vol 1. L'attachement, Paris, PUF. 
Brubaker Rogers, 2001. Au-delà de l'identité, Actes de la recherche en sciences sociales 139: L'exception américaine, pp. 66-85.

CaIllé Alain, 2007. Anthropologie du don, Paris, La Découverte.

Camour Luc Enoka, 2010. Lien et séparation familiale de l'enfant kanak dans le monde socioculturel de Kaala-Gomen, Pouembout, Éditions de la province Nord.

Ciccone Albert, 2001. Enveloppe psychique et fonction contenante: modèles et pratiques, Cabiers de psychologie clinique 17, pp. 81-102 (https://www.cairn.info/load_pdf.php?ID_ ARTICLE=CPC_017_0081).

Cugola Umberto, 2009. Les contradictions du développement. La tribu de La Conception à Nouméa, thèse de doctorat, Toulouse, Université Jean-Jaurès.

_, 2017 (à paraître). De la contrainte judiciaire à l'étreinte éducative : la voie étroite, Journal $d u$ droit des jeunes.

Devereux Georges, 1977 (3'ème éd.). Essai d'ethnopsychiatrie générale, Paris, Gallimard.

Dufour Dany-Robert, 2012. Le divin Marché. La révolution culturelle libérale, Paris, Gallimard.

—, 2015. Lindividu Qui vient... après le libéralisme, Paris, Gallimard.

—, 2016. La situation désespérée du temps présent me remplit d'espoir, Lormont, Éd. Bord de l'eau.

Elias Norbert, 1991. La Société des individus, Paris, Fayard.

FAnon Frantz, 1971 (1 ${ }^{\text {̀re }}$ éd. 1952). Peau noire masques blancs, Paris, Seuil.

Foucault Michel, 2011. Surveiller et punir. Naissance de la prison, Paris, Gallimard.

Gabarron-Garcia Florent, 2016. L'inconscient, c'est la politique (Deleuze aujourd'hui), entretien avec J.-P. Cazier, Diacritik (https:// diacritik.com/2016/01/27/florent-gabarrongarcia-linconscient-cest-la-politique-deleuzeaujourdhui/).

Gauchet Marcel, 2012. Pour une théorie psychanalytique de l'individuation, in Karl-Léo Schwering (éd.), Se construire comme sujet, entre fliation et sexuation, Toulouse, ERES.

Gori Rolland. La fabrique des imposteurs. Babel, Paris, 2015,

Green André, 2010. Hommage à Bernard Juillerat. Un interlocuteur des psychanalystes, Journal de la Société des Océanistes 130-131: Hommage à Bernard Juillerat, pp. 65-66 (https://jso.revues.org/6073).
Houseman Michael et Carlos Severi, 1994. Naven ou le donner à voir. Essai d'interprétation de l'action rituelle, Paris, CNRs Éditions.

Juillerat Bernard, 2005. CR de Mouchenik Yoram, L'enfant vulnérable. Psychothérapie transculturelle en pays kanak, Gradhiva, pp. 151-152 (http://gradhiva.revues.org/439).

Karsz Saul, 2014. Mythe de la parentalité, réalités familiales, Paris, Dunod.

Kohler Jean-Marie, Patrick Pillon et Loïc J.D. WACQUANT, 1985. Jeunesse canaque et coutume, Nouméa, ORSTOM, Institut culturel mélanésien.

La BoÉtie Étienne (de), 1922. Discours de la servitude volontaire, Bossard.

LaCAN Jacques, 1967 (séance du 10 mai). La logique du fantasme. Séminaire 1966-1967, Paris, Édition de l'Association lacanienne internationale.

Laplantine François, 1972. L'ethnopsychiatrie, Paris, Éd. universitaires.

—, 2007. Le sujet. Essai d'anthropologie politique, Paris, Tétraèdre.

Lasch Christopher, 2000. La culture du narcissisme, Paris, Flammarion, Champs.

Leblic Isabelle, 1993. Les Kanak face au développement. La voie étroite, Grenoble, PUG avec le soutien de l'ADCK.

Lebreton David, 2014. Adolescence et conduites à risque, Bruxelles, Éditions Fabert, coll. Temps d'arrêt.

Lebrun Jean-Pierre, 2007. La perversion ordinaire, Paris, Denoël.

Lyotard Jean-François, 1979. La condition postmoderne. Rapport sur le savoir, Paris, Les éditions de Minuit.

Mead Margaret, 1963. Mours et sexualité en Océanie, Paris, Plon.

Melman Charles, 2009. La nouvelle économie psychique: la façon de penser et de jouir aujourd'hui, Toulouse, ERES.

Monnerie Denis et Pierre Lemonnier, 2010. Bernard Juillerat. Une passion du sujet, Journal de la Société des Océanistes 130-131: Hommage à Bernard Juillerat, pp. 5-20 (https://jso. revues.org/6175).

Monnerie Denis, 2012. Objets cérémoniels et élaborations des relations sociales (Arama et Hoot ma Whaap, Kanaky Nouvelle-Calédonie, Mélanésie), Techniques \& culture 58, pp. 121-141. 
Moucheniк Yoram, 2004. L'enfant vulnérable. Psychothérapie transculturelle en pays kanak, Paris, La Pensée sauvage.

Mucchielli Laurent, 2014. Sociologie de la délinquance, Paris, Armand Colin.

Nathan Tobie, 1986. La folie des autres. Traité d'ethnopsychiatrie clinique, Paris, Dunod.

SAuret Marie-Jean, 1993. La psychologie clinique, histoire et discours. De l'intérêt de la psychanalyse, Toulouse, PUM.
Van Gennep Arnold, i98I. Les rites de passage, Paris, Nourry.

Winnicott Donald, 1969. De la pédiatrie à la psychanalyse, Paris, Рвр.

Zafiropoulos Markos, 2003. Lacan et LéviStrauss ou le retour à Freud, 1951-1957, Paris, PUF, coll. Philosophie d'aujourd'hui.

_, 2015. Le symptôme et l'esprit du temps, Paris, PUF, coll. Essai. 